\title{
Physical Activity and Social Cognition in the Elderly
}

\author{
Juan José Alarcón-Jiménez ${ }^{1}$, Adriana Nielsen-Rodríguez ${ }^{1}{ }^{\mathbb{D}}$, Ramón Romance ${ }^{1, * \mathbb{C}}$, \\ Ricardo Gómez-Huelgas ${ }^{2,3}$ and M. Rosa Bernal-López ${ }^{2,3}$ \\ 1 Human Kinetics and Body Composition Laboratory, Department of Didactics of Languages, Arts and Sports, \\ Universidad de Málaga, 29010 Málaga, Spain; juanconor91@hotmail.com (J.J.A.-J.); \\ adriananielsen@uma.es (A.N.-R.) \\ 2 Internal Medicine Department, Regional University Hospital of Malaga, Instituto de Investigación Biomédica de \\ Málaga (IBIMA), Universidad de Málaga, 29010 Málaga, Spain; ricardogomezhuelgas@hotmail.com (R.G.-H.); \\ robelopajiju@yahoo.es (M.R.B.-L.) \\ 3 CIBER Fisiopatología de la Obesidad y la Nutrición, Instituto de Salud Carlos III, 28029 Madrid, Spain \\ * Correspondence: arromance@uma.es; Tel.: +34-952132454
}

Received: 29 April 2020; Accepted: 5 June 2020; Published: 8 June 2020

\begin{abstract}
Social cognition is related to the so-called "Theory of the mind" and refers to the ability to understand the behavior of other people. It plays a transcendental role in the social relations cohesion. Knowing whether normal aging is associated with deficiencies in social understanding is a problem in characterizing the changes that occur in adulthood. The aim of this study was to examine whether the social cognition of the elderly is mediated by factors such as working memory, physical activity, body composition, or age, in elderly people. Sixty volunteers (mean age, $71.50 \pm 4.84$ years) were recruited from various locations in Malaga. Recognition of emotional expression, working memory, body composition, and physical activity were evaluated. Our results show that performance in emotion recognition is not affected by age in the range studied. Furthermore, we have not found a relationship with any of the body composition parameters. However, a significant relationship with performance in working memory has been revealed, as well as a significant relationship between vigorous/very vigorous physical activity and working memory. Therefore, physical activity could be positively related to the recognition of emotions by positively influencing working memory.
\end{abstract}

Keywords: Theory of Mind; social intelligence; exercise; body composition; working memory; older adults

\section{Introduction}

"Social Intelligence" or "Social Cognition" are terms directly related to the so-called "Theory of Mind" (ToM) and refer to the ability to understand and predict the behavior of other people, their knowledge, their intentions, their emotions, and their beliefs [1].

Consequently, ToM is a distinctive capacity that underlies social interaction and allows us to represent the mental states of others. Therefore, it plays a transcendental role in cohesion of individual and social relationships [2]. Furthermore, for humans, social interaction development could represent the last step in the evolution of our cognitive architectures [3].

Initially, the study of ToM was limited to primatology and generalized developmental disorders along with acquired brain and cognitive disorders related to aging [4]. However, social cognition is a complex process in which there are mechanisms to perceive, process, and evaluate stimuli, which allows us to represent the social environment $[3,5]$. Thereby, when we refer to ToM, we talk about a complex system of metacognitive skills directed at knowing the contents of the cognitive system of others [6]. Generally, these skills are studied and evaluated at various levels: first- and second-order beliefs, social 
use of language, social behavior and empathy, and facial recognition of emotions and decoding of mental states only from information of the eyes.

In the present study, we focused on facial recognition of emotions and decoding of mental states only from information of the eyes. Recognition of facial emotions is the ability to identify and recognize basic forms of affective and emotional expression by observing people's faces [7]. Emotional facial recognition is affected by age, context, and some characteristics of cognitive and neuronal development, and the incorrect interpretation of emotions is related to interpersonal and social problems, low social functioning, and inappropriate social behaviors $[8,9]$.

Within this set of complex metacognitive skills, emotional expression is studied through the gaze. When we interact with others, we are constantly modulating our message or our actions according to their facial expression, and we also use the movements and factions of our face, trying to emphasize what we say or execute. Therefore, the ability to read or interpret the others' eyes, or "read the mind in the eyes" (RME), is a key component in the study and evaluation of ToM [10].

Although some studies have already used RME task to demonstrate differences in performance in certain population profiles [11,12], there is little evidence of studies assessing social cognition and its relationship with other variables in adults with normal intelligence [10], and therefore it is necessary to carry out studies such as this one to fill the gap in the literature.

\subsection{ToM and its Relation with Other Cognitive Abilities}

As we have seen in previous paragraphs, ToM plays a fundamental role in the cohesion of individual and social relationships. Some authors suggested that this mechanism may be innate and managed in a genetically determined module that is activated by environmental stimuli [13], and it would consist of a specific and specialized cognitive domain for the understanding of mental states [1]. This particularity would make it possible for this function to be damaged in a person with normal functionality in other aspects.

An alternative hypothesis, supported by injury studies and some work in autism, is that ToM is based on metarepresentation. In this sense, it is thought that there is a relationship between ToM and other cognitive skills related to reasoning abilities and understanding of mental states [14]. Besides, ToM could be related to executive functions, since it presents a cognitive component related to them as working memory or planning processes, and other more emotional components related to moral dilemmas [6]. Likewise, there could be a close relationship between the execution of tasks related to the evaluation of ToM and working memory, since the difficulties in registering, updating, maintaining, or inhibiting information of patients with a severe impairment of the central executive system cannot solve this type of task [6].

\subsection{ToM and Aging}

Knowing if normal aging is associated with impairments in social understanding is a critical issue to characterize the changes that occur in adulthood. Skills related to ToM are acquired with age forming a kind of social wisdom in each person, and some methodological advances show that ToM works independently of general cognition in aging, but there is currently a great debate about ToM as a special cognitive module $[6,12,14]$. The first investigations carried out in this regard supported the general hypothesis that ToM is not affected by age, but in recent years the results show evidence that there are specific measurable deficits in ToM with increasing age [15].

The effects of Parkinson's disease on ToM were also investigated, and additional evidence was obtained suggesting that ToM is not affected by aging [16]. Other authors found similar performance in healthy older and younger participants in several different ToM paradigms, reporting that the elderly were not affected in a ToM task and that there are no exclusive impediments for them $[4,8]$.

Finally, studies of the effects of Alzheimer's disease and dementia on ToM found that there were no significant effects of age on ToM dysfunction, despite both disorders being related to ToM deficits. However, ToM impairment was significantly more severe in dementia than in Alzheimer's disease 
and, in turn, ToM deficits were much less severe than general cognitive dysfunction in Alzheimer's disease [17].

\subsection{Physical Activity as a Stimulus-Generating Element for the Formation of ToM}

As ToM is a capacity developed with brain maturation and activated by environmental stimuli that can be developed in different ways, physical activity (PA) can be understood as a vehicle that allows varied social experiences and, therefore, the formation and maintenance of social cognition. Moreover, since we are born, movement allows us contact and relationship with our environment and with others. Furthermore, PA can induce acute and chronic effects on structural and functional levels in the aging brain and produces beneficial effects on our cognition that contribute to the maintenance of physical well-being and a healthy cognitive state that allows us the performance of daily activities [18-24].

Every day, new benefits that physical exercise brings to our physical and mental health are discovered. The effects that PA has on the functionality and structure of our nervous system are increasingly evident, and the findings that relate the state of physical fitness with executive functions [25,26], memory [27,28], and mental flexibility [26] are increasingly consistent. However, few studies have analyzed the effects that PA has on social cognition.

In view of the above, we can say that the study of the relationship between social cognition and PA in healthy elderly has been scarce. The aim of the present study was to examine whether the social cognition, indexed in facial recognition of emotions and working memory of healthy elderly people, is mediated by various factors such as the amount and intensity of PA performed, body composition, or age in elderly people.

\section{Materials and Methods}

\subsection{Participants}

Sixty female volunteers (mean age, $71.50 \pm 4.84$ ) were recruited for this study. The recruitment process was carried out in the healthy aging centers and in the Sport Preventive Medicine area in Málaga city (Andalusia, Spain), between September 2019 and February 2020. Once the possible candidates were identified, we proceeded to contact them by phone to explain the design and objectives of the study and invite them to participate.

After obtaining written informed consent, the subjects underwent a clinical interview by a doctor of internal medicine, nurse, psychologist, nutritionist, and physical activity monitor. Exclusion criteria included a self-reported history of psychiatric illness or any other serious medical condition (e.g., diabetes, cardiovascular disease). Of the 99 people who agreed to participate, 33 were excluded from the study for not meeting the established inclusion criteria, leaving a sample of 60 women and 6 men. Due to the low number of men, which did not constitute a representative sample, we decided to exclude them and carry out the study only with women.

This study, conducted from the Regional University Hospital of Malaga, was developed following the ethical guidelines of the World Medical Association Declaration of Helsinki (WMA 2013) and has the approval of the Malaga Provincial Research Ethics Committee of the Andalusian Government Health Department.

\subsection{Instruments}

To assess recognition of emotional expression through the gaze, participants carried out the Reading the Mind in the Eyes test (RMET) of Baron-Cohen et al. [10]. This assessment instrument is widely used in research studies with various disorders [29]. A translation and adaptation to Spanish, retrieved from the Autism Research Center of the University of Cambridge, was used [30].

The test consists of a total of 36 photographs that were exposed on a monitor. Each of them were accompanied by four adjectives from which the participant must choose only one of them (the one considered to correspond more accurately to the image). The selected word was circled on a 
paper records sheet. The subject was warned that there was no time limit, but should do so in the shortest possible time. If any of the terms were not understood, the help manual could be consulted. A difficulty weighted score (RMETdiff) of the photographs was calculated based on performance norms of the test manual. We understood as the easiest photographs those with the lowest number of incorrect responses, while the most difficult were those with the highest number of incorrect responses. Then, more difficult photographs received a higher weighting than the easier ones [10].

To assess working memory and information processing, participants completed the "Digits tasks" and "Letters and Numbers" task from the Spanish adaptation of the Wechsler Adult Intelligence Scale-Fourth Edition (WAIS-IV) [31]. The tasks were performed according to the instructions provided by the test manual. The internal consistency of the Spanish adaptation of the WAIS-IV has been studied using the two-halves method and the Cronbach's alpha coefficient, except for the Symbol Search, Number Keys, and Cancellation tests, which used the test-retest correlation. The reliability coefficients vary slightly according to the age group and range between 0.72 and 0.93 for the tasks and between 0.87 and 0.97 for the Indices and the Total Intelligence Quotient (TIQ). The correlations between the different tasks on the scale, for all age groups, range between 0.12 (Cancellation-Comprehension) and 0.64 (Digits-Letters and numbers) [31].

To estimate the amount and intensity of PA, ActiGraph wGT3X-BT ${ }^{\circledR}$ accelerometers (ActiGraph LLC, Pensacola, FL, USA) were used for 7 days. Accelerometers are commonly used for assessing PA in humans and, more concretely, ActiGraph wGT3X-BT ${ }^{\circledR}$ accelerometers have been widely used in studies [32]. The accelerometers were placed on the wrist of the nondominant hand and an epoch of $60 \mathrm{~s}$ was established. The cut points used were from Freedson VM3 (2011): Light 0 to 2690, Moderate 2690 to 6166, Vigorous 6166 to 9642, Very vigorous 9643 and above, MVPA Minimum Count 2690 [33-36].

Total body composition was estimated using a Hologic QDR $4500^{\circledR}$ dual-energy x-ray absorptiometry (DXA) scanner (Hologic Inc., Bedford, MA, USA), which is a gold standard for body composition estimation $[37,38]$. Each subject was scanned by a certified technician, and the distinguished bone and soft tissue, edge detection, and regional demarcations were done by computer algorithms with APEX Software 3.0 ${ }^{\circledR}$ (APEX Corporation Software, Pittsburg, PA, USA). For each scan, subjects wore sport clothes and were asked to remove all materials that could attenuate the X-ray beam, including jewelry items. Calibration of the densitometer was checked daily against standard calibration block supplied by the manufacturer.

\subsection{Procedure}

All participants were tested in the morning (10:00-12:00 h). Participants first completed the RMET as indicated on performance norms of the test manual. This test requires participants to recognize different mental states from photographs that show only the eye area of the face and indicate one of the four adjectives presented with the image shown. Then, participants had a five-minute break before completing the aforementioned WAIS-IV tasks according to the instructions provided by the test manual.

Once this was done, participants were scanned to determine their body composition using the Hologic QDR $4500^{\circledR}$ dual-energy x-ray absorptiometry (DXA) scanner and following the procedure explained above. Finally, participants were provided with the accelerometer they would carry for 7 days. It was placed on the wrist of the nondominant hand and, after a week, the accelerometer was returned for data extraction.

\subsection{Data Analysis}

After the normality analysis of the variables, the correlation coefficients were calculated taking into account their parametric or nonparametric nature.

Later, to determine whether some relationships between RMETdiff and very vigorous PA (VVPA) were present, a linear regression was performed to assess the ability of VVPA to predict performance on the RMETdiff. Furthermore, to determine whether a relationship between working memory and 
RMETdiff was present, working memory was entered into a simple regression as a predictor of RMETdiff. Then, a third linear regression was performed to determine the relationship between VVPA and working memory. Finally, a multiple regression was performed with the variables that had shown a relationship with RMETdiff.

Analyses were conducted to ensure the assumptions of normality, linearity, multicollinearity, and homoscedasticity were not violated.

\section{Results}

Descriptive statistics are presented in Table 1 and bivariate correlation coefficients (CI $=95 \%)$ among measures are presented in Table 2.

Table 1. Descriptive statistics.

\begin{tabular}{ccc}
\hline Variable & Mean & SD \\
\hline Age & 71.40 & 4.94 \\
WM & 17.01 & 3.37 \\
RMET DIFF & 33.70 & 7.49 \\
LPA & 705.40 & 139.07 \\
MPA & 287.27 & 79.70 \\
VPA & 65.29 & 48.44 \\
VVPA & 7.27 & 8.65 \\
MVPA & 359.83 & 107.43 \\
LEAN & $38,457.09$ & 4921.54 \\
MASS & $71,258.48$ & 9697.72 \\
\%FAT & 45.78 & 4.78 \\
BMD & 0.81 & 0.08 \\
\hline
\end{tabular}

Age (years); WM: working memory (obtained score); RMET DIFF: reading the mind in the eyes test weighted for difficulty (obtained score); LPA:, Light Physical Activity (minutes/week); MPA: Moderate Physical Activity (minutes/week); VPA: Vigorous Physical Activity (minutes/week); VVPA: Very Vigorous Physical Activity (minutes/week); MVPA: Moderate and Vigorous Physical Activity (minutes/week); LEAN: Lean Mass (Kg); MASS (Kg); \%FAT; BMD: Bone Mineral Density $\left(\mathrm{g} / \mathrm{cm}^{2}\right)$.

Table 2. Simple correlation coefficients among measures $(\mathrm{N}=60)$.

\begin{tabular}{|c|c|c|c|c|c|c|c|c|c|c|c|}
\hline & Age & WM & RMET DIFF & LPA & MPA & VPA & VVPA & MVPA & LEAN & MASS & $\%$ FAT \\
\hline WM & -0.131 & & & & & & & & & & \\
\hline $\begin{array}{l}\text { RMET } \\
\text { DIFF }\end{array}$ & 0.038 & $0.659^{* *}$ & & & & & & & & & \\
\hline LPA & 0.164 & -0.111 & -0.123 & & & & & & & & \\
\hline MPA & -0.140 & -0.039 & 0.056 & $-0.726^{* *}$ & & & & & & & \\
\hline VPA & -0.496 ** & 0.158 & 0.118 & $-0.475^{* *}$ & $0.321^{*}$ & & & & & & \\
\hline VVPA & $-0.355 * *$ & $0.214 *$ & 0.134 & $-0.257^{*}$ & 0.134 & 0.639 ** & & & & & \\
\hline MVPA & $-0.329^{*}$ & 0.182 & 0.124 & $-0.755^{* *}$ & $0.868^{* *}$ & $0.731^{* *}$ & $0.532 * *$ & & & & \\
\hline LEAN & -0.213 & 0.002 & -0.054 & 0.018 & 0.060 & 0.106 & 0.063 & 0.137 & & & \\
\hline MASS & $-0.376^{* *}$ & 0.061 & 0.074 & 0.108 & -0.093 & 0.257 & 0.200 & 0.056 & 0.712 ** & & \\
\hline$\%$ FAT & $-0.285^{*}$ & 0.126 & 0.139 & 0.156 & -0.222 & 0.168 & 0.131 & -0.109 & -0.157 & $0.423 * *$ & \\
\hline BMD & $-0.323 *$ & -0.036 & -0.069 & -0.119 & 0.148 & 0.268 & 0.247 & 0.262 & $0.569^{* *}$ & $0.504 * *$ & -0.203 \\
\hline
\end{tabular}

${ }^{* *} p<0.01,{ }^{*} p<0.05$. Age (years); WM: working memory (obtained score); RMET DIFF: reading the mind in the eyes test weighted for difficulty (obtained score); LPA:, Light Physical Activity (minutes/week); MPA: Moderate Physical Activity (minutes/week); VPA: Vigorous Physical Activity (minutes/week); VVPA: Very Vigorous Physical Activity (minutes/week); MVPA: Moderate and Vigorous Physical Activity (minutes/week); LEAN: Lean Mass (Kg); MASS (Kg); \%FAT; BMD: Bone Mineral Density $\left(\mathrm{g} / \mathrm{cm}^{2}\right)$.

Regarding age, no significant relationship was found with either RMETdiff $(\mathrm{r}=0.38, p>0.05)$ or working memory (rho $=-0.131, p>0.05$ ).

Obviously, a significant negative relationship was found between age and the amount and intensity of physical activity performed by the subjects VPA (rho $=-0.492, p<0.01$ ), VVPA (rho $=-0.355$, $p<0.01)$, MVPA ( $\mathrm{r}=-0.329, p<0.05)$. Likewise, a negative and significant relationship was found between Age and Mass $(\mathrm{r}=-0.376, p<0.01)$, BMD $(\mathrm{r}=-0.323, p<0.05)$ and \%FAT $(\mathrm{r}=-0.285, p<0.05)$ since, as we reach advanced ages, the body tissues become lighter. 
RMETdiff was positively related to working memory (rho $=0.659, p<0.01$ ), but no relationship was found between RMETdiff or any of the variables of PA. However, working memory was positively related to VVPA (tau_b $=0.214, p<0.05$ ).

A linear regression was performed first to assess the ability of VVPA to predict performance on the RMETdiff. The predictor VVPA ( $\mathrm{B}=0.249, \mathrm{~B}$ SE $=0.109, \beta=0.288, \mathrm{t}=2.288,95 \% \mathrm{CI}[0.031,0.467])$ significantly accounted for $8.3 \%\left(95 \% \mathrm{CI}\right.$ for $\mathrm{R}^{2}$; adjusted $\left.\mathrm{R}^{2}=0.067\right)$ of the variance on RMETdiff performance, $\mathrm{F}(1,58)=5.237, p=0.026$. Another linear regression was carried out to assess the ability of working memory to predict performance on the RMETdiff. The predictor working memory $(B=1.005, B$ SE $=0.153, \beta=0.653, t=6.562,95 \% C I[0.70,1.31])$ significantly accounted for $42.6 \%(95 \%$ CI for $\mathrm{R}^{2}$; adjusted $\left.\mathrm{R}^{2}=0.42\right)$ of the variance on RMETdiff performance, $\mathrm{F}(1,58)=43.055, p=0.000$. A third linear regression was performed to assess the ability of VVPA to predict performance on working memory. The predictor VVPA $(B=0.196, \mathrm{~B} \mathrm{SE}=0.069, \beta=0.349, \mathrm{t}=2.836,95 \%$ CI [0.058, $0.335])$ significantly accounted for $12.2 \%\left(95 \% \mathrm{CI}\right.$ for $\mathrm{R}^{2}$; adjusted $\left.\mathrm{R}^{2}=0.11\right)$ of the variance on working memory performance, $\mathrm{F}(1,58)=8.040, p=0.006$.

Finally, a multiple regression (Table 3) was implemented to study the possible relationship between the predictor variables (age, working memory, and VVPA) and RMETdiff. The variables (Age, working memory, and VVPA) significantly predicted a total of $45.8 \%\left(95 \%\right.$ CI for $\mathrm{R}^{2}$; adjusted $\left.\mathrm{R}^{2}=0.429\right)$ of the variance in RMETdiff, $F(1,56)=37.483, p=0.000$. Only working memory presented a significant relationship ( $\mathrm{B}=0.966, p<0.01)$, Age $(\mathrm{B}=0.264, p=0.094)$ and VVPA $(\mathrm{B}=0.092, p=0.327)$ being excluded from the model due to their low relevance.

Table 3. Multiple regression analysis for variables predicting RMETdiff.

\begin{tabular}{cccc}
\hline Variable & B & B SE & $\boldsymbol{\beta}$ \\
\hline Age & 0.264 & 0.155 & 0.174 \\
WM & 0.966 & 0.162 & $0.645^{* *}$ \\
VVPA & 0.092 & 0.093 & 0.106 \\
\hline
\end{tabular}

** $p<0.01$. Age (years); WM: working memory (obtained score); VVPA: Very Vigorous Physical Activity (minutes/week).

\section{Discussion and Conclusions}

This study aimed to help the understanding of the influence that some factors have on social cognition of the elderly indexed in the facial recognition of emotions. For this, a series of elements that can influence were controlled, including age, body composition (lean mass, fat mass, and bone mineral density), amount and intensity of PA, and working memory.

It seems logical to think that, if our brain is affected by aging, ToM could also be affected by the appearance of impairments in cognitive operations. Two interesting possibilities regarding the relationship between ToM and aging can be discussed: on the one hand, if ToM is linked to the functioning of a specific cognitive architecture, the declines in that cognitive architecture can also be associated with declines in social functioning. For example, if the elderly show a detriment in "fluid" intelligence, a worse performance in ToM could be explained by a lower capacity of fluency that makes it difficult for them to work, and not by a specific domain problem in ToM [4,39]. On the other hand, the accumulation of knowledge about the social world throughout one's life can lead us to be much more efficient in our social interactions, and maybe for that the elderly show a conserved capacity of ToM that does not deteriorate with aging.

Some studies have already used RMET trying to demonstrate differences in performance in certain population profiles [11,12], pointing out that the performance obtained in this test by the elderly was lower than that obtained by young people [4,40-42] and that the difficulties in recognition are probably independent of the decreases in general cognitive declines [2]. Bailey et al. [41] added that the reduction of cognitive empathy was associated with less social participation in older participants. 
Slessor et al. [43] reported this deterioration in elderly people, but it also occurred in the control conditions and the authors suggested that the deficiencies observed in ToM could produce general deficiencies in social understanding due to advanced age. For their part, Duval et al. [44] also reported that older participants were affected when decoding complex mental states, but not to recognize basic emotions, and that the effect of age on RME task performance was not mediated by processing speed, executive function, or episodic memory.

As a result of these studies that use the RME task in aging, we could say that, in general, this capacity is affected by age. However, in light of the results obtained in our study, we can say that performance in the facial recognition of emotions is not affected by age in the age range studied (60-88 years). These results contradict the studies that used RME to report its relationship with aging and that indicated that this ability is affected by age, although in those cases, the analyzed age ranges were different [4]. Therefore, our data reveals that elderly people show a preserved ToM capacity that does not decrease with aging in the studied age range.

These results support the data obtained in other studies that suggest that the difference between the young and old group is nonexistent or not significant $[15,16,41,45-47]$, and some of them even indicated that the elderly tend to perform quite well on tasks that assess ToM. In fact, Nolaker et al. [15] reported that the elderly participants performed significantly better than younger participants in some ToM tasks. These findings suggest that social cognition is a domain in which experience must be taken into account.

Nevertheless, our findings are also in line with other subsequent research that does not exactly share the idea that ToM tasks are not specifically affected in the elderly [15]. There are variables related to general cognitive abilities, such as crystallized and fluid intelligence and working memory, that could alter the conclusions. In fact, today there is an active debate about the relationship between social cognition and other cognitive abilities.

Regarding the relationship between facial recognition of emotions and other cognitive abilities, some authors reported that, in most cases, facial recognition of emotions is independent of general cognitive declines [2], suggesting that this mechanism may be innate and specific [1,13]. However, an alternative hypothesis is that ToM is based on meta-representation [14], and therefore ToM could be related to executive functions such as working memory or planning processes, as well as another more emotional components related to moral dilemmas [6].

Baksh et al. [4] made a difference between executive functions, working memory and decision-making tasks, and emotional recognition. These authors concluded by stating that, in normal aging, there is an affectation of the first functions and not of the latter ones. Subsequent research reveals age-related deficits that are likely to depend in whole or in part on general skills such as working memory, processing speed, and executive function [2]. Furthermore, although memory and the ability to retrieve verbal and nonverbal material is probably one of the first cognitive functions to suffer deterioration due to age $[48,49]$, in our sample we have found that performance on working memory test is not affected by age in the studied interval (60-88 years).

In another study [50], three groups (young, middle-aged, and elderly) were compared under conditions that varied in the requirement to recall key information. The two groups of older participants had worse results under a load of memory, which suggests a direct effect of working memory on ToM performance.

In our case, the results have shown a significant relationship between performance in working memory tests and RMETdiff, specifically the predictor working memory significantly accounted for $42.6 \%$ of the variance on RMETdiff performance. These results support the theory that there could be a close relationship between the execution of tasks related to ToM assessment and working memory due to the need to record, update, and maintain information [6].

Our findings are also in accordance with other studies in which groups of elderly participants performed worse under memory load, suggesting a direct effect for working memory on ToM 
performance $[2,6,50]$. Thus, performance in facial recognition of emotions probably depends partially or totally on general skills such as working memory, processing speed, and executive function.

Given that ToM would be a capacity that can be acquired in different ways and activated by environmental stimuli [13], PA could constitute an excellent vehicle that allows varied social experiences and, therefore, the formation and maintenance of social cognition. Furthermore, knowing that PA produces beneficial effects on our body, our nervous system, and our cognition [22], it seems logical to think that these effects would be equally beneficial in social cognition, understood as a cognitive ability.

In other words, social cognition could benefit, on the one hand, from healthy effects that physical exercise brings to our nervous system and, on the other hand, from the increase in experiences and social contacts derived of the practice of PA, and both factors would affect the greater development and/or maintenance of social competence [51-55]. Similarly, some factors such as diseases and possible consequent pain can determine the amount of PA that one person can carry out, and therefore their social contact amount [56].

Several studies have shown that physically active people have a reduced risk of cognitive impairment and dementia $[57,58]$. The PA that we can perform on a daily basis, even without being of great intensity, could favor cognitive maintenance and delay its impairment. This was reported by Kattenstroth et al. [59], who performed a six-month dance intervention and claimed that even moderate levels of PA, in combination with a rich sensorimotor and cognitive compromise, can help alleviate age-related deterioration.

In the present study, based on the results obtained, we have found a significant relationship between RMETdiff and the amount of VVPA (attributable to healthy elderly people). The predictor VVPA significantly accounted for $8.3 \%$ of the variance on RMETdiff performance. In addition, a relationship between working memory and VVPA was found; in our case, the predictor VVPA significantly accounted for $12.2 \%$ of the variance on working memory performance. However, we have not obtained any significant relationship between RMETdiff and other amounts or types of intensity of PA, taking into account the cutoff points used. There were also no relationships between RMETdiff with any of the body composition parameters evaluated.

These results are partially in line with those of Ruscheweyh et al. [60], who evaluated healthy elderly individuals while participating in a six-month intervention of PA of medium or low intensity. These authors reported that increases in total PA were positively associated with an increase in memory. Thus, there is a correlation between the increase in total PA and better episodic memory in elderly individuals after low and medium intense physical training.

There are some limitations to the study. First, the fact that the sample is formed only by women can influence the results obtained. In addition, some variables such as the level of studies or the socioeconomic level of the subjects have not been taken into account, despite the fact that these data could be important in the results obtained in the tests carried out and in the cognitive performance of the participants in the study.

Future work lines would benefit from increase of the sample, since increasing the number of subjects evaluated and including both men and women in a similar proportion could help to obtain more representative results of the studied reality. Another option could be to extend the age range covered or establish two age groups to compare the results between older and younger people. In the same way, it could be interesting to design a more ambitious longitudinal study that followed up on a group of subjects over the years to check the influence of the aging process on cognitive skills and social cognition. It could also be considered the possibility of applying to the data of the amount and intensity of PA other cut points that are also valid for our age range, verifying if new correlations are obtained between the variables studied. Finally, although in this study we have focused on working memory, there are other executive functions that can influence facial recognition of emotions, and whose study can be very interesting to understand ToM functioning.

In summary, although a consensus on whether ToM is an innate or acquired ability has not yet been reached in the literature, there is no doubt that it is a specific cognitive domain differentiated from 
the others, managed by a genetically determined module that is activated by environmental stimuli [13]. That is, ToM is a capacity that can be influenced by external factors such as PA, which is known that it can induce acute and permanent changes in structural and functional levels in the aging brain.

The discovered benefits of PA on physical and mental health are increasing and, as other cognitive abilities are improved thanks to exercise, the healthy effects that PA brings to our nervous system could benefit ToM so much, in part because of the increase in experiences and social contacts that involves a greater amount of physical activity [53-55].

Furthermore, the PA that we carry out daily, regardless of its intensity, produces physiological effects that favor the maintenance of a healthy mental and cognitive state, delaying its deterioration [59]. The PA that we can carry out daily could favor maintenance and delay cognitive deterioration and even more if it is carried out in combination with a sensorimotor and cognitive compromise, being able to help alleviate age-related deterioration [59].

In light of the results obtained, we conclude that ToM is not directly influenced by age, which was proven thanks to the performance of the elderly in facial recognition of emotions tasks. However, it is affected in some way by the decline suffered by some executive functions due to the aging process, and at the same time, is benefited by the practice of PA.

Furthermore, our results show that working memory is not negatively affected by age but does benefit from PA, preferably of vigorous or very vigorous intensity. Therefore, we can affirm that PA has certain direct effects on social cognition, but it also has indirect effects, since it improves working memory and this, in turn, also influences the best performance in RMETdiff.

These findings encourage us to continue investigating to determine which cognitive functions are related to ToM and what variables can influence both its detriment and its improvement, and from there, we can formulate effective recommendations that contribute to the maintenance and the improvement of people's cognitive health and social cognition.

In any case, what we already know about the benefits that PA has on the brain and its functions, including social cognition, is more than enough to demonstrate the need to promote PA among the population, considering that it depends on our physical, cognitive and social well-being.

Author Contributions: All authors contributed to the outline of the proposal and the paper design. J.J.A.-J. and A.N.-R. wrote the paper, with significant input and critical revisions from the other authors; A.N.-R. and R.R. collected body composition and cognitive data; R.R. was responsible for data processing and analysis, and revised and edited the text; and R.G.-H. and M.R.B.-L. contributed by funding acquisition, project administration and conducting hospital tests on subjects. All authors read and approved the final manuscript.

Funding: This research was funded by Universidad de Málaga (Spain) and by grants from the Instituto de Salud Carlos III, cofinanced by the Fondo Europeo de Desarrollo Regional-FEDER (PI18/00766 and "Centros de Investigación En Red" [CIBER, CB06/03/0018]). M. Rosa Bernal-Lopez was supported by the "Miguel Servet Type I" program (CP15/00028) from the ISCIII-Madrid (Spain), cofinanced by the Fondo Europeo de Desarrollo Regional-FEDER. Adriana Nielsen-Rodríguez was supported by "Formación de Profesorado Universitario" (FPU17/01554) grants from the Ministry of Education, Culture and Sport (Spain).

Conflicts of Interest: The authors declare no conflict of interest and the funders had no role in the design of the study; in the collection, analyses, or interpretation of data; in the writing of the manuscript, or in the decision to publish the results.

\section{References}

1. Schaafsma, S.M.; Pfaff, D.W.; Spunt, R.P.; Adolphs, R. Deconstructing and reconstructing theory of mind. Trends Cogn. Sci. Regul. Ed. 2015, 19, 65-72. [CrossRef]

2. Moran, J.M. Lifespan development: The effects of typical aging on theory of mind. Behav. Brain Res. 2013, 237, 32-40. [CrossRef]

3. Estes, D.; Bartsch, K. Theory of mind: A foundational component of human general intelligence. Behav. Brain Sci. 2017, 40, e201. [CrossRef]

4. Baksh, R.A.; Abrahams, S.; Auyeung, B.; MacPherson, S.E. The Edinburgh Social Cognition Test (ESCoT): Examining the effects of age on a new measure of theory of mind and social norm understanding. PLoS ONE 2018, 13. [CrossRef] 
5. Spunt, R.P.; Adolphs, R. The neuroscience of understanding the emotions of others. Neurosci. Lett. 2019, 693, 44-48. [CrossRef]

6. Tirapu-Ustarroz, J.; Perez-Sayes, G.; Erekatxo-Bilbao, M.; Pelegrin-Valero, C. What is theory of mind? Rev. Neurol. 2007, 44, 479-489. [PubMed]

7. Ekman, P.; Cordaro, D. What is Meant by Calling Emotions Basic. Emot. Rev. 2011, 3, 364-370. [CrossRef]

8. Arango de Montis, I.; Bruene, M.; Fresan, A.; Ortega Font, V.; Villanueva, J.; Saracco, R.; Muñoz-Delgado, J. Recognition of facial expression of the emotions and their relation to attachment styles and psychiatric symptoms. Preliminary study on Psychiatric Residents. Salud Ment. 2013, 36, 95-100. [CrossRef]

9. Ruffman, T.; Henry, J.D.; Livingstone, V.; Phillips, L.H. A meta-analytic review of emotion recognition and aging: Implications for neuropsychological models of aging. Neurosci. Biobehav. Rev. 2008, 32, 863-881. [CrossRef]

10. Baron-Cohen, S.; Wheelwright, S.; Hill, J.; Raste, Y.; Plumb, I. The "Reading the Mind in the Eyes" test revised version: A study with normal adults, and adults with Asperger syndrome or high-functioning autism. J. Child Psychol. Psychiatry 2001, 42, 241-251. [CrossRef]

11. Richell, R.A.; Mitchell, D.G.V.; Newman, C.; Leonard, A.; Baron-Cohen, S.; Blair, R.J.R. Theory of mind and psychopathy: Can psychopathic individuals read the 'language of the eyes'? Neuropsychologia 2003, 41, 523-526. [CrossRef]

12. Dodell-Feder, D.; Ressler, K.J.; Germine, L.T. Social cognition or social class and culture? On the interpretation of differences in social cognitive performance. Psychol. Med. 2020, 133-145. [CrossRef] [PubMed]

13. Scholl, B.J.; Leslie, A.M. Minds, modules, and meta-analysis. Child Dev. 2001, 72, 696-701. [CrossRef] [PubMed]

14. Stone, V.E.; Gerrans, P. What's domain-specific about theory of mind? Soc. Neurosci. 2006, 1, 309-319. [CrossRef] [PubMed]

15. Nolaker, E.J.; Murray, K.; Happe, F.; Charlton, R.A. Cognitive and Affective Associations with an Ecologically Valid Test of Theory of Mind across the Lifespan. Neuropsychology 2018, 32, 754-763. [CrossRef] [PubMed]

16. Saltzman, J.; Strauss, E.; Hunter, M.; Archibald, S. Theory of mind and executive functions in normal human aging and Parkinson's disease. J. Int. Neuropsychol. Soc. 2000, 6, 781-788. [CrossRef]

17. Bora, E.; Walterfang, M.; Velakoulis, D. Theory of mind in behavioural-variant frontotemporal dementia and Alzheimer's disease: A meta-analysis. J. Neurol. Neurosurg. Psychiatry 2015, 86, 714-719. [CrossRef]

18. Charness, N. Aging and human performance. Hum. Factors 2008, 50, 548-555. [CrossRef]

19. Heyn, P.C.; Johnson, K.E.; Kramer, A.F. Endurance and strength training outcomes on cognitively impaired and cognitively intact older adults: A meta-analysis. J. Nutr. Health Aging. 2008, 12, 401-409. [CrossRef]

20. Bamidis, P.D.; Vivas, A.B.; Styliadis, C.; Frantzidis, C.; Klados, M.; Schlee, W.; Siountas, A.; Papageorgiou, S.G. A review of physical and cognitive interventions in aging. Neurosci. Biobehav. Rev. 2014, 44, 206-220. [CrossRef]

21. Drag, L.L.; Bieliauskas, L.A. Contemporary review 2009: Cognitive aging. J. Geriatr. Psychiatry Neurol. 2010, 23, 75-93. [CrossRef] [PubMed]

22. Erickson, K.I.; Kramer, A.F. Aerobic exercise effects on cognitive and neural plasticity in older adults. Br. J. Sports Med. 2009, 43, 22-24. [CrossRef] [PubMed]

23. Erickson, K.I.; Voss, M.W.; Prakash, R.S.; Basak, C.; Szabo, A.; Chaddock, L.; Kim, J.S.; Heo, S.; Alves, H.; White, S.M. Exercise training increases size of hippocampus and improves memory. Proc. Natl. Acad. Sci. USA 2011, 108, 3017-3022. [CrossRef] [PubMed]

24. Voelcker-Rehage, C.; Godde, B.; Staudinger, U.M. Physical and motor fitness are both related to cognition in old age. Eur. J. Neurosci. 2010, 31, 167-176. [CrossRef] [PubMed]

25. Kramer, A.F.; Colcombe, S.J. Fitness Effects on the Cognitive Function of Older Adults: A Meta-Analytic Study-Revisited. Perspect. Psychol. Sci. 2018, 13, 213-217. [CrossRef]

26. Gordon, B.A.; Rykhlevskaia, E.I.; Brumback, C.R.; Lee, Y.; Elavsky, S.; Konopack, J.F.; McAuley, E.; Kramer, A.F.; Colcombe, S.; Gratton, G.; et al. Neuroanatomical correlates of aging, cardiopulmonary fitness level, and education. Psychophysiology 2008, 45, 825-838. [CrossRef]

27. Hindin, S.B.; Zelinski, E.M. Extended Practice and Aerobic Exercise Interventions Benefit Untrained Cognitive Outcomes in Older Adults: A Meta-Analysis. J. Am. Geriatr. Soc. 2012, 60, 136-141. [CrossRef] 
28. Liu-Ambrose, T.; Nagamatsu, L.S.; Graf, P.; Beattie, B.L.; Ashe, M.C.; Handy, T.C. Resistance training and executive functions: A 12-month randomized controlled trial. Arch. Intern. Med. 2010, 170, 170-178. [CrossRef]

29. Martin-Contero, M.C.; Secades-Villa, R.; Aparicio-Migueza, A.; Tirapu-Ustarroz, J. Empathy in severe mental disorders. Rev. Neurol. 2017, 64, 145-152. [CrossRef] [PubMed]

30. ARC Tests. Available online: https://www.autismresearchcentre.com/arc_tests (accessed on 14 September 2019).

31. Wechsler, D. WAIS-IV: Escala de Inteligencia de Wechsler para Adultos-IV; Pearson: Madrid, Spain, 2012.

32. Santos-Lozano, A.; Marín, P.J.; Torres-Luque, G.; Ruiz, J.R.; Lucía, A.; Garatachea, N. Technical variability of the GT3X accelerometer. Med. Eng. Phys. 2012, 34, 787-790. [CrossRef] [PubMed]

33. Matthews, C.E.; Hagströmer, M.; Pober, D.M.; Bowles, H.R. Best practices for using physical activity monitors in population-based research. Med. Sci. Sports Exerc. 2012, 44 (Suppl. 1), S68-S76. [CrossRef] [PubMed]

34. Trost, S.G.; McIver, K.L.; Pate, R.R. Conducting Accelerometer-Based Activity Assessments in Field-Based Research. Med. Sci. Sports Exerc. 2005, 37, S531-S543. [CrossRef] [PubMed]

35. Plasqui, G.; Bonomi, A.; Westerterp, K. Daily physical activity assessment with accelerometers: New insights and validation studies. Obes. Rev. 2013, 14, 451-462. [CrossRef] [PubMed]

36. Sasaki, J.E.; John, D.; Freedson, P.S. Validation and comparison of ActiGraph activity monitors. J. Sci. Med. Sport 2011, 14, 411-416. [CrossRef] [PubMed]

37. Scafoglieri, A.; Clarys, J.P. Dual energy X-ray absorptiometry: Gold standard for muscle mass? J. Cachexia Sarcopenia Muscle 2018, 9, 786-787. [CrossRef]

38. Kim, J.; Wang, Z.; Heymsfield, S.B.; Baumgartner, R.N.; Gallagher, D. Total-body skeletal muscle mass: Estimation by a new dual-energy X-ray absorptiometry method. Am. J. Clin. Nutr. 2002, 76, 378-383. [CrossRef]

39. Hedden, T.; Gabrieli, J.D.E. Insights into the ageing mind: A view from cognitive neuroscience. Nat. Rev. Neurosci. 2004, 5, 87-96. [CrossRef]

40. Phillips, L.H.; MacLean, R.D.J.; Allen, R. Age and the understanding of emotions: Neuropsychological and sociocognitive perspectives. J. Gerontol. B Psychol. Sci. Soc. Sci. 2002, 57, P526-P530. [CrossRef]

41. Bailey, P.E.; Henry, J.D. Growing less empathic with age: Disinhibition of the self-perspective. J. Gerontol. $B$ Psychol. Sci. Soc. Sci. 2008, 63, P219-P226. [CrossRef]

42. Pardini, M.; Nichelli, P.F. Age-Related Decline in Mentalizing Skills Across Adult Life Span. Exp. Aging Res. 2009, 35, 98-106. [CrossRef]

43. Slessor, G.; Phillips, L.H.; Bull, R. Exploring the specificity of age-related differences in theory of mind tasks. Psychol. Aging. 2007, 22, 639-643. [CrossRef] [PubMed]

44. Duval, C.; Piolino, P.; Bejanin, A.; Eustache, F.; Desgranges, B. Age effects on different components of theory of mind. Conscious. Cogn. 2011, 20, 627-642. [CrossRef] [PubMed]

45. Bailey, P.E.; Henry, J.D.; Von Hippel, W. Empathy and social functioning in late adulthood. Aging Ment. Health 2008, 12, 499-503. [CrossRef] [PubMed]

46. Li, X.; Wang, K.; Wang, F.; Tao, Q.; Xie, Y.; Cheng, Q. Aging of theory of mind: The influence of educational level and cognitive processing. Int. J. Psychol. 2013, 48, 715-727. [CrossRef]

47. Castelli, I.; Baglio, F.; Blasi, V.; Alberoni, M.; Falini, A.; Liverta-Sempio, O.; Nemni, R.; Marchetti, A. Effects of aging on mindreading ability through the eyes: An fMRI study. Neuropsychologia 2010, 48, 2586-2594. [CrossRef]

48. Grady, C.L.; Springer, M.V.; Hongwanishkul, D.; McIntosh, A.R.; Winocur, G. Age-related changes in brain activity across the adult lifespan. J. Cogn. Neurosci. 2006, 18, 227-241. [CrossRef]

49. Grady, C. Brain ageing. The cognitive neuroscience of ageing. Nat. Rev. Neurosci. 2012, 13, 491-505. [CrossRef]

50. Pezzuti, L.; Longobardi, E.; Milletti, K.; Ovidi, A. A study about the Theory of Mind in primary and secondary aging. Life Span Disabil. 2011, 14, 31-44.

51. Strachan, S.M.; Brawley, L.R.; Spink, K.; Glazebrook, K. Older adults' physically-active identity: Relationships between social cognitions, physical activity and satisfaction with life. Psychol. Sport Exerc. 2010, 11, 114-121. [CrossRef]

52. Quintana, D.S.; Guastella, A.J.; Outhred, T.; Hickie, I.B.; Kemp, A.H. Heart rate variability is associated with emotion recognition: Direct evidence for a relationship between the autonomic nervous system and social cognition. Int. J. Psychophysiol. 2012, 86, 168-172. [CrossRef] 
53. Mortimer, J.A.; Ding, D.; Borenstein, A.R.; DeCarli, C.; Guo, Q.H.; Wu, Y.G.; Zhao, Q.; Chu, S. Changes in Brain Volume and Cognition in a Randomized Trial of Exercise and Social Interaction in a Community-Based Sample of Non-Demented Chinese Elders. J. Alzheimers Dis. 2012, 30, 757-766. [CrossRef] [PubMed]

54. Merom, D.; Cumming, R.; Mathieu, E.; Anstey, K.J.; Rissel, C.; Simpson, J.M.; Morton, R.L.; Cerin, E.; Sherrington, C.; Lord, S.R. Can social dancing prevent falls in older adults? a protocol of the Dance, Aging, Cognition, Economics (DAnCE) fall prevention randomised controlled trial. BMC Public Health 2013, $13,477$. [CrossRef] [PubMed]

55. Soubelet, A. The Role of Social Activity in Age-Cognition Relations. Educ. Gerontol. 2013, 39, 558-568. [CrossRef]

56. Gyurcsik, N.C.; Brawley, L.R.; Spink, K.S.; Glazebrook, K.E.; Anderson, T.J. Is Level of Pain Acceptance Differentially Related to Social Cognitions and Behavior? The Case of Active Women with Arthritis. J. Health Psychol. 2011, 16, 530-539. [CrossRef] [PubMed]

57. Sofi, F.; Valecchi, D.; Bacci, D.; Abbate, R.; Gensini, G.F.; Casini, A.; Macchi, C. Physical activity and risk of cognitive decline: A meta-analysis of prospective studies. J. Intern. Med. 2011, 269, 107-117. [CrossRef] [PubMed]

58. Hamer, M.; Chida, Y. Physical activity and risk of neurodegenerative disease: A systematic review of prospective evidence. Psychol. Med. 2009, 39, 3-11. [CrossRef]

59. Kattenstroth, J.C.; Kalisch, T.; Holt, S.; Tegenthoff, M.; Dinse, H.R. Six months of dance intervention enhances postural, sensorimotor, and cognitive performance in elderly without affecting cardio-respiratory functions. Front. Aging Neurosci. 2013, 5, 5. [CrossRef]

60. Ruscheweyh, R.; Willemer, C.; Krueger, K.; Duning, T.; Warnecke, T.; Sommer, J.; Völker, K.; Ho, H.V.; Mooren, F.; Knecht, S.; et al. Physical activity and memory functions: An interventional study. Neurobiol. Aging 2011, 32, 1304-1319. [CrossRef]

(C) 2020 by the authors. Licensee MDPI, Basel, Switzerland. This article is an open access article distributed under the terms and conditions of the Creative Commons Attribution (CC BY) license (http://creativecommons.org/licenses/by/4.0/). 